\title{
Análisis de los argumentos elaborados por estudiantes de cursos introductorios de Física universitaria ante situaciones problemáticas
}

\section{Analysis of the arguments created by students in introductory courses of university Physics when tackling problematic situations}

\author{
Mikel Ceberio, José Manuel Almudí, José Luis Zubimendi \\ Departamento de Física Aplicada I, Euskal Herriko Unibertsitatea (UPV/EHU) \\ mikel.ceberio@ehu.es; josemanuel.almudi@ehu.es; jl.zubimendi@ehu.es
}

RESUMEN • En este artículo analizamos la calidad de los argumentos utilizados por los estudiantes universitarios que siguen una enseñanza tradicional en primer curso de Ingeniería cuando, ante una situación problemática de física, deben optar por la estrategia o vía de resolución más adecuada. Valoramos lo apropiado de sus conclusiones, la pertinencia de las leyes y principios que han considerado para llegar a una conclusión (pertinencia conceptual), la calidad de las pruebas manejadas y si sus conclusiones se encuentran validadas por la coherencia de los razonamientos (suficiencia del razonamiento). Las deficiencias más destacables han surgido ligadas a la ausencia de pruebas que sustenten la conclusión y al hecho de que, cuando se consideran estas pruebas, a menudo no se razona adecuadamente la relación entre ellas y las conclusiones.

PALABRAS CLAVE: argumentación; resolución de problemas; enseñanza de la Física en la universidad.

ABSTRACT - In this article, we analyse the quality of the arguments used by the college students who follow a traditional teaching in first engineering course when, faced with a problematic situation of physics, must choose by the strategy or most appropriate resolution path. We value the appropriateness of their conclusions, the pertinence of the laws and principles that have been considered to reach a conclusion (conceptual pertinence), the quality of the evidence managed and if its conclusions are validated by the consistency of reasoning (sufficiency of the reasoning). The most noteworthy shortcomings have arisen relating to the lack of evidence supporting the explanation and the fact that, when considering this evidence, often, it is not properly reasoned the relationship between the evidence and the conclusions.

KEYWORDS: argumentation; problem solving; Physics teaching at university.

Fecha de recepción: febrero 2013 • Aceptado: junio 2013 


\section{INTRODUCCIÓN}

Tradicionalmente, los objetivos de aprendizaje de las ciencias, en general, y de la física, en particular, se han focalizado fundamentalmente en el desarrollo de un conocimiento conceptual en relación con los contenidos, las leyes y los principios propios de la materia. Sin embargo, la investigación en didáctica de las ciencias (Driver et al., 2000; Duschl y Osborne, 2002; Duschl, 2008) defiende que la instrucción en el aula debe desplazarse desde modelos que enfatizan la transmisión del conocimiento del profesor al alumno, hacia modelos que acentúen la construcción del conocimiento y su validación por medio de la investigación. Las nuevas orientaciones en el ámbito de la enseñanza de las ciencias (Pellegrino et al., 2002; Sawyer, 2006) destacan la importancia de que los estudiantes sean capaces de plantear formas complejas de razonamiento similares a las propias de la actividad científica.

Así mismo, existe un amplio consenso entre los epistemólogos e investigadores (Chalmers, 2000; Duschl, 2000) en cuanto a que, generalmente, dicha investigación científica parte de situaciones problemáticas abiertas (y no de "datos puros»), para cuya solución se deberán elaborar «tentativas de respuesta», en términos de hipótesis basadas en el cuerpo de conocimiento que, posteriormente, deberán ser contrastadas. Estas hipótesis y los diseños para su contrastación son los que guían la búsqueda de los datos. La investigación científica, por tanto, puede entenderse como un proceso de construcción por medio del cual se producen teorías explicativas que den respuesta a las observaciones y a los datos y que deben ser expuestas a la revisión, debate y crítica por parte de la comunidad científica (Driver et al., 2000; Duschl, 2000; Sandoval y Reiser, 2004). En consecuencia, la capacidad para elaborar un argumento claro y convincente que coordine los datos o pruebas (evidence en inglés) y la teoría, para defender o refutar un planteamiento, constituye un aspecto fundamental de la investigación científica (Driver et al., 2000; Duschl y Osborne, 2002; Jiménez-Aleixandre et al., 2000). En este sentido, Kuhn (2005) establece como objetivo clave de la educación científica el desarrollo de formas de pensamiento para las que la investigación y la argumentación resultan ser aspectos centrales.

En los últimos años, un creciente número de investigaciones están centrando su atención en el análisis de la argumentación en las clases de ciencias. La investigación ha abordado cómo los estudiantes articulan y justifican sus conclusiones o explicaciones (entre otros, Bell, 2004; Lawson, 2002; Sandoval y Millwood, 2005; Zohar y Nemet, 2002), así como el proceso por medio del cual los estudiantes interaccionan entre ellos cuando proponen, evalúan y critican ideas (entre otros, Abell et al., 2000; Clark y Sampson, 2006; Kuhn y Reiser, 2006; Kuhn y Udell, 2003; Osborne et al., 2004).

El análisis de los argumentos elaborados por los estudiantes puede aportar información importante acerca de la comprensión de los contenidos científicos alcanzada (por ejemplo, las teorías, leyes y principios de la física), el razonamiento científico de los estudiantes, los aspectos epistemológicos (por ejemplo, qué cuenta como justificación en ciencias) y la habilidad de los estudiantes para comunicar y justificar ideas.

Por otro lado, los resultados de la investigación en resolución de problemas han mostrado los límites de la enseñanza tradicional en este ámbito. La preocupación por el pobre aprendizaje de los estudiantes universitarios en resolución de problemas, así como la escasa comprensión de conceptos y leyes de la física, ha llevado al desarrollo de diferentes planteamientos de enseñanza de la resolución de problemas (Ceberio et al., 2008; Martínez Torregrosa, 2005). Cuando los estudiantes se enfrentan a problemas de lápiz y papel, un aspecto ineludible de cara a la resolución lo constituye la identificación de las leyes y principios fundamentales de la física aplicables en ese contexto. Resulta necesario, así mismo, ante una situación problemática determinada, valorar posibles vías alternativas de resolución. La elección de una u otra pasa por una adecuada justificación basada en pruebas o evidencias.

En este artículo, pretendemos analizar la calidad de los argumentos utilizados por los estudiantes que siguen una enseñanza tradicional en primer curso de Ingeniería cuando, ante una situación 
problemática de física, deben optar por la estrategia o vía de resolución más adecuada. Tratamos de comprobar si sus conclusiones, en este sentido, se encuentran validadas por la coherencia de sus razonamientos según el análisis de las interacciones características de esa situación y que constituirán las pruebas del argumento.

\section{MARCO TEÓRICO}

La investigación se ha apoyado en un amplio rango de perspectivas analíticas para examinar la naturaleza y la calidad de los argumentos generados por los estudiantes en el ámbito de la enseńanza de las ciencias. Tal y como señalan Sampson y Clark (2008), tres parecen ser los aspectos críticos para los investigadores que estudian el modo en que los estudiantes generan argumentos en el contexto de las ciencias: los componentes de un argumento, lo adecuados que resultan los diversos componentes desde la perspectiva de su contenido científico y la naturaleza de la justificación (cómo las conclusiones son validadas en el argumento). Muchos de los modelos teóricos de análisis utilizados por los investigadores describen un argumento en términos de conclusión y justificación basado en pruebas. Si bien se utilizan también términos distintos como aseveración o explicación, en la mayoría de los modelos se considera un elemento equivalente a la conclusión que requiere justificación.

Una parte muy significativa de la literatura sobre argumentación en enseñanza de las ciencias se ha fundamentado en el trabajo de Toulmin (1958). La concepción de argumento de este autor ha permitido a los investigadores examinar la calidad de los argumentos en una gran variedad de dominios, entre los cuales se encuentra la ciencia (Erduran et al., 2004; Jiménez-Aleixandre et al., 2000; Osborne et al., 2004).

En el modelo de argumento de Toulmin (conocido como Toulmin's Argument Pattern, TAP) (figura 1), surgen interconectados la conclusión, los datos que soportan la conclusión, la justificación que aporta el nexo entre los datos y la conclusión, los calificadores modales que expresan el grado de certeza del argumento y el conocimiento básico que respalda la justificación. Finalmente, las condiciones de refutación indicarían las circunstancias en las cuales las justificaciones perderían su validez. De acuerdo con Toulmin, la validez de un argumento debe estar sustentada, necesariamente, por la coherencia de su justificación.

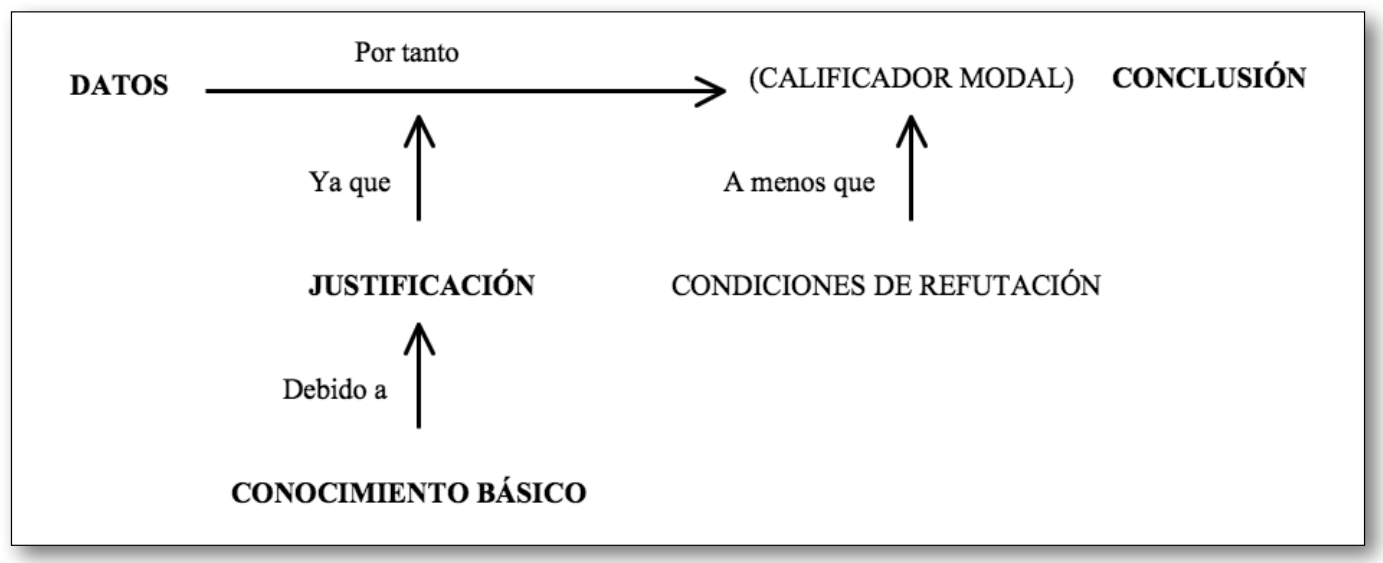

Fig. 1. Modelo de Toulmin (TAP).

Sampson y Clark (2009) plantean un modelo de análisis de la calidad de los argumentos inspirado en el modelo de Toulmin y en consonancia con otros investigadores en enseñanza de las ciencias (por ejemplo, Kuhn y Reiser, 2005; McNeill y Krajcik, 2007; Osborne et al., 2004). En dicho modelo (una 
adaptación del cual se representa en la figura 2), se considera que un argumento científico está constituido por tres componentes interrelacionados: la conclusión o explicación (similar a la conclusión de Toulmin), la prueba (similar a los datos de Toulmin) y el razonamiento (una combinación de la justificación y el conocimiento básico de Toulmin). La explicación o conclusión (que, en adelante, esta última forma de denominarla será la única que utilicemos), en función de lo que la situación demande, puede consistir, entre otros, en la solución a un problema, articular una relación descriptiva entre variables o enunciar un mecanismo causal. La prueba, de origen teórico o empírico, requiere que los estudiantes consideren medidas, observaciones, señales o motivos que soporten la validez o legitimidad de la conclusión. Para que esta información pueda ser considerada como una prueba debe ofrecer, para mostrar una evolución en el tiempo, una diferencia entre grupos o una relación entre variables. Ha de ser relevante en la justificación de la conclusión y específica para la cuestión discutida (Kenyon et al., 2006). El razonamiento debe mostrar por qué la prueba soporta la conclusión y por qué la prueba puede considerarse como tal. El razonamiento descrito en este modelo coincidiría básicamente con lo que otros teóricos e investigadores denominan «esquemas de los argumentos» (Perelman, 1969; van Eemeren y Grootendorst, 2004; Walton, 2008) que permiten la conexión entre lo que denominan premisas y la conclusión o tesis del argumento.

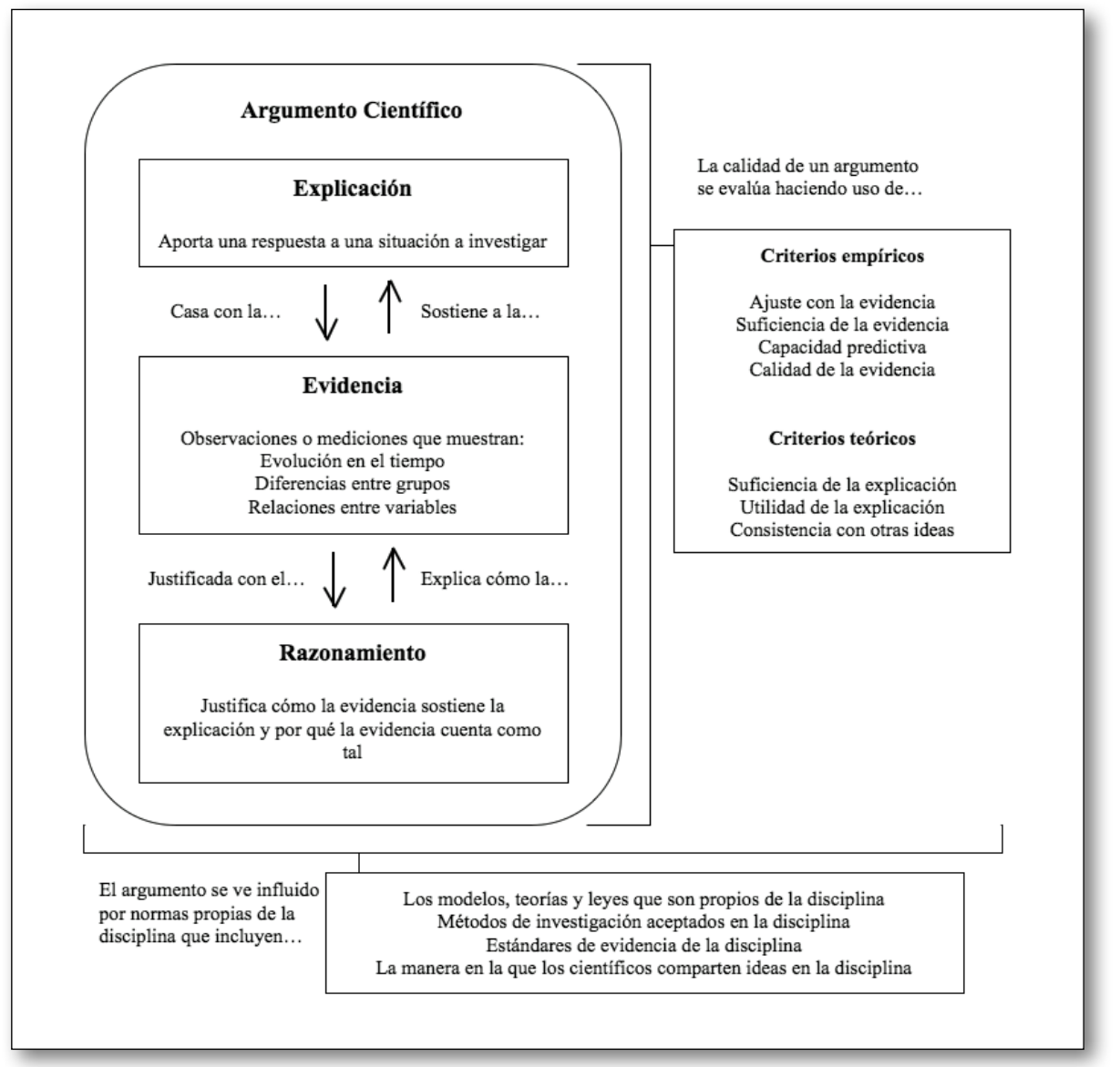

Fig. 2. Modelo de Sampson y Clark.

Este modelo de Sampson y Clark, además de recoger cuáles son los componentes de un argumento, aporta a estudiantes e investigadores criterios específicos para evaluar la calidad de un argumento en ciencia. De acuerdo con los autores, este planteamiento se ha mostrado como una manera adecuada y productiva tanto para introducir a los estudiantes en la compleja tarea de generar argumentos, como 
para evaluar la calidad de los argumentos que construyen. En este sentido, la calidad de un argumento es evaluada considerando criterios empíricos y criterios teóricos. En los primeros se tiene en cuenta cómo concuerda la conclusión con la prueba, la capacidad predictiva de la conclusión, la suficiencia de la prueba mencionada y su calidad. En los criterios teóricos se considera la consistencia de la conclusión con el conocimiento teórico y la utilidad de esta para comprender la cuestión planteada.

Concretamente, los autores (Sampson et al., 2011) focalizan su atención en los principales aspectos que la literatura utiliza para evaluar la calidad de los argumentos escritos empleados por los estudiantes, esto es: $a$ ) lo apropiado de conclusión, que debe ser correcta; $b$ ) la pertinencia de los referentes conceptuales considerados en el argumento, que deben relacionarse con las leyes y principios de la materia; c) la calidad de la prueba, que tiene que ser adecuada y suficiente, y d) la suficiencia del razonamiento, que debe justificar que la prueba soporta la conclusión.

Estos cuatro aspectos, con especial énfasis en la suficiencia del razonamiento, han sido, así mismo, nuestra referencia para el análisis de los argumentos construidos por nuestros estudiantes cuando se han enfrentado a la elección de las vías de resolución de una situación problemática y que a continuación describimos.

\section{METODOLOGÍA}

La investigación se realizó con estudiantes de primer curso del Grado en Ingeniería de la Universidad del País Vasco. La totalidad de los estudiantes habían cursado con anterioridad dos años de estudios de física en el bachillerato (16-18 años) y abordaban su primer curso de física para ingenieros.

La asignación de los estudiantes a los diferentes grupos se efectuó de forma aleatoria por medio de una aplicación informática. Para cada uno de los idiomas en los que se imparte la docencia, castellano o euskera, el único criterio utilizado es el de igualdad del número de estudiantes por grupo. En estos grupos se encuentran mezclados estudiantes del Grado en Ingeniería Eléctrica, Grado en Ingeniería Electrónica Industrial y Automática y Grado en Ingeniería Mecánica, puesto que estas titulaciones comparten un tronco común en primero y segundo cursos.

Estos estudiantes recibieron 2 horas de clase magistral, 1 hora de clase de problemas y 2 horas de laboratorio o seminario (alternativamente) por semana, durante 15 semanas en el área de mecánica (primer semestre), y otras 15 semanas en el área de electromagnetismo (segundo semestre). Las clases las impartieron profesores del departamento de Física con amplia experiencia docente e investigadora y que, en todos los casos, han superado al menos una prueba de selección pública para ser miembros de plantilla de la Universidad del País Vasco. Los estudiantes utilizaron los libros de texto habituales para las clases magistrales y para la resolución de problemas de final del capítulo, abarcando el mismo programa para todos ellos.

En el estudio que se describe en este artículo tomaron parte un número total de 153 estudiantes de primer curso de Ingeniería distribuidos en 2 clases con entre 80 y 90 estudiantes por clase magistral, entre 40 y 45 en clase de problemas y entre 20 y 25 en seminarios y laboratorio. El porcentaje de alumnos que repetía curso era del $10 \%$.

Para recabar datos, hemos pedido a los estudiantes que argumenten justificadamente, de manera individual y por escrito, la vía de resolución que consideran más adecuada para resolver una determinada situación problemática (cuadros 1 y 2). Las situaciones I y II se contextualizan en el ámbito de la cinemática, la dinámica y el principio de conservación de la energía mecánica. Las situaciones III y IV se relacionan con la aplicabilidad de los principios de conservación del momento lineal y del momento angular. Todas ellas, por tanto, pertenecen al ámbito de la mecánica y pueden considerarse aspectos estándar de la materia. En los propios enunciados se incluyen preguntas relativas al dominio específico de las situaciones planteadas que buscan orientar las respuestas de los estudiantes hacia los aspectos más generales de un argumento científico: la conclusión, la prueba y el razonamiento. 
Cuadro 1.

Situaciones problemáticas propuestas I y II

Situación I) Un mono se deja caer libremente desde lo alto de un puente que une las orillas de un río. Situación II) Ese mismo mono se deja caer desde el puente pero agarrado al extremo libre de una cuerda elástica similar a un muelle. El otro extremo de la cuerda elástica se encuentra unido a la barandilla del puente y su longitud natural coincide con la altura de la barandilla, de manera que cuando el mono se suspende de la cuerda esta comienza a estirarse. La elasticidad de la cuerda permite que el mono alcance el agua agarrado a ella.

Con objeto de obtener la velocidad con la que el mono llega al río en cada una de las situaciones anteriores, I y II, indica, sin resolver matemáticamente:

a) ¿Qué estrategia de resolución (qué ley, qué principio, qué fórmula) usarías para obtener la velocidad?

b) Si además del modo propuesto en el apartado anterior existe, o no, otra posible alternativa.

c) ¿Qué características de las interacciones (fuerzas) que actúan sobre el mono, en cada caso, son las que valoras para elegir un camino de resolución?

d) ¿Por qué, según las características de las interacciones (fuerzas), propones uno o más caminos de resolución y descartas otros?

La situación I, teniendo en cuenta que la única fuerza que realiza trabajo, el peso, es conservativa, se podrá resolver por aplicación de la conservación de la energía mecánica. Por otro lado, como el peso se mantiene constante durante la caída, la aplicación de la segunda ley de Newton, junto con las ecuaciones de la cinemática correspondientes al movimiento uniformemente acelerado, nos permitiría, de una manera sencilla, obtener la velocidad. En la situación II, las fuerzas que realizan trabajo, peso y fuerza elástica, también son conservativas, por lo que la vía energética es igualmente válida. En este caso, sin embargo, la fuerza elástica varía con la posición, por lo que el movimiento resultante no es uniformemente acelerado y la vía cinemática resultaría más compleja (prácticamente descartable para los estudiantes).

\section{Cuadro 2.}

Situaciones problemáticas propuestas III y IV

Situación III) Sobre la superficie de una pista de hielo sin rozamiento se encuentra en reposo, en posición horizontal, un bastón de hockey. En un momento dado, una pelota de plastilina lanzada por un jugador choca perpendicularmente contra el bastón en su extremo recto y queda incrustada en él. Situación IV) Una pequeña puerta de rejilla metálica que sirve de cierre de la pista de hielo se encuentra parcialmente abierta. En un momento dado, la pelota de plastilina lanzada por un jugador choca perpendicularmente contra la puerta en su vértice inferior, en la posición más alejada de las bisagras y queda incrustada en ella.

Con objeto de obtener la velocidad con la que el sistema bastón-pelota, en la situación III, y el sistema puerta metálica-pelota, en la situación IV, se mueven tras la colisión, indica, sin resolver matemáticamente:

a) ¿Qué estrategia/s de resolución (qué ley/es, qué principio/s) usarías para obtener la velocidad?

b) ¿Qué características de las interacciones (fuerzas) que actúan sobre el sistema son las que valoras para elegir un camino de resolución?

c) ¿Por qué, sobre la base de las características de las interacciones (fuerzas), propones uno u otro camino de resolución? 
El movimiento del bastón tras la colisión descrita en la situación III podrá ser estudiado considerando la conservación del momento lineal y la conservación del momento angular en el choque. Esto es posible porque, durante el breve intervalo de tiempo que dura la colisión, la resultante de las fuerzas externas es nula (peso y normal se compensan entre sí y no existe rozamiento). Las interacciones entre el bastón y la pelota de plastilina son fuerzas internas al sistema considerado, por lo que no hacen variar el momento lineal. Sin embargo, en la situación IV, no será posible aplicar el principio de conservación del momento lineal puesto que, durante el choque, actúan fuerzas externas sobre el sistema (las ejercidas por las bisagras sujetas a la pared) que no se pueden considerar despreciables frente a las internas y que impiden la traslación de dicho sistema. Como consecuencia de que estas fuerzas externas se apliquen en el mismo eje de rotación que pasa por las bisagras, el momento creado por ellas con respecto a ese eje es nulo, y por tanto el momento angular respecto a él sí se conservará durante el choque. En ambas situaciones se descarta la vía energética por tratarse de un choque real inelástico.

Inspirados en el planteamiento de Sampson et al. (2011), para cada uno de los cuatro aspectos de referencia que empleamos con objeto de medir la calidad de los argumentos construidos por los estudiantes, y que adelantábamos en el apartado 2, marco teórico (lo apropiado de la conclusión, la pertinencia de las referencias conceptuales consideradas, la calidad de la prueba y la suficiencia del razonamiento), planteamos un sistema de puntuación en escala de 0 a 2 , en función de unos determinados parámetros específicos para cada situación problemática (tablas 1, 2, 3 y 4). Posteriormente, las puntuaciones obtenidas en los cuatro aspectos anteriores se combinan para dar lugar a la puntuación que corresponde al argumento emitido. Como resultado, a cada argumento le corresponde un valor comprendido entre $0 \mathrm{y}$ 8, de tal manera que los marcadores más elevados representan argumentos de mayor calidad.

En las situaciones analizadas, la conclusión apropiada se corresponde con la adecuada elección de las leyes y principios de la física aplicables en la resolución. Respondería, por tanto, a la pregunta «qué» estrategia/s de resolución son aplicable/s para resolver la situación problemática. La pertinencia conceptual del argumento refleja si para llegar a la conclusión se han tenido en cuenta las leyes y los principios que potencialmente son aplicables, independientemente de que la elección (conclusión) sea apropiada o no lo sea. Es decir, podría ser pertinente valorar la aplicabilidad de determinadas leyes y principios y, por tanto, el argumento ser conceptualmente pertinente y, sin embargo, la elección de la ley o principio ser inapropiada y, en consecuencia, la conclusión no ser correcta. Así, por ejemplo, en la situación II (caída bajo la acción de una fuerza elástica), sería conceptualmente pertinente valorar la posibilidad de aplicar la vía cinemático-dinámica; por el contrario, su elección como vía de resolución sería inapropiada (conclusión inapropiada).

En cada situación planteada se describe un proceso observable (caída libre, caída bajo la acción de una fuerza elástica y, por tanto, más lentamente que en la caída libre, choque plástico sin eje fijo, que da lugar a una traslación y a una rotación, y choque plástico con eje fijo, que únicamente da lugar a una rotación). La observación de cada proceso (dado el contexto de las situaciones abordadas y el tipo de preguntas que en ellas se plantean) en sí misma no constituye una prueba que nos sirva para llegar de una forma razonada a la conclusión (elección de la vía de resolución). La prueba, de carácter teórico, del argumento que permitirá elegir entre las posibles opciones de resolución está implícita en la situación descrita y se corresponde con las interacciones (las fuerzas) y sus características (constantes, variables con la posición, conservativas o no conservativas, internas o externas). La búsqueda de la prueba demanda, por tanto, un análisis cualitativo de las interacciones en cada situación.

Finalmente, en el razonamiento se debe aportar un enunciado que, combinando justificación y conocimiento básico acerca de las leyes y principios y sus condiciones de aplicabilidad, ponga en relación la conclusión (elección de la vía de resolución) con las pruebas (características de las interacciones). Respondería, por tanto, a la pregunta de "por qué» la/s estrategia/s de resolución propuesta/s son aplicable/s para resolver la situación problemática (o "por qué» no lo son las que se descartan). 
Bajo este enfoque, un buen argumento estará constituido por un razonamiento suficiente que conecte justificadamente las pruebas (características de las interacciones) con las conclusiones (estrategias de resolución), considerando para ello el conocimiento conceptual pertinente (leyes, principios y sus condiciones de aplicabilidad).

Tabla 1.

Criterios específicos de valoración de la calidad

de los argumentos correspondientes a la situación problemática I

\begin{tabular}{|c|c|c|}
\hline \multicolumn{3}{|r|}{ Situación $I$} \\
\hline \multirow{3}{*}{$\begin{array}{l}\text { Conclusión } \\
\text { apropiada }\end{array}$} & 2 & $\begin{array}{l}\text { Proponer que es posible utilizar tanto el principio de conservación de la energía mecánica como } \\
\text { la segunda ley de Newton junto con la cinemática del movimiento rectilíneo y uniformemente } \\
\text { acelerado. }\end{array}$ \\
\hline & 1 & Proponer utilizar solo una de las dos vías posibles señaladas en el nivel 2. \\
\hline & 0 & Proponer utilizar otra vía de resolución distinta de las anteriores o no proponer ninguna. \\
\hline \multirow{3}{*}{$\begin{array}{l}\text { Pertinencia } \\
\text { conceptual }\end{array}$} & 2 & $\begin{array}{l}\text { Considerar la posibilidad de aplicar el principio de conservación de la energía así como la se- } \\
\text { gunda ley de Newton y las leyes cinemáticas (con independencia de la propuesta final). }\end{array}$ \\
\hline & 1 & $\begin{array}{l}\text { Considerar la posibilidad de aplicar el principio de conservación de la energía o la posibilidad } \\
\text { de aplicar la segunda ley de Newton y leyes cinemáticas (con independencia de la propuesta } \\
\text { final). }\end{array}$ \\
\hline & 0 & No considerar la posibilidad de aplicar ninguna de las leyes o principios anteriores. \\
\hline \multirow{3}{*}{$\begin{array}{l}\text { Calidad } \\
\text { de la prueba }\end{array}$} & 2 & $\begin{array}{l}\text { Indicar todas las fuerzas que actúan sobre el mono. Distinguir cuáles son conservativas y cuáles } \\
\text { no lo son. Distinguir cuáles son constantes durante el desplazamiento y cuáles no lo son. }\end{array}$ \\
\hline & 1 & $\begin{array}{l}\text { Indicar todas las fuerzas que actúan sobre el mono. Distinguir cuáles son conservativas y cuáles } \\
\text { no lo son o distinguir cuáles son constantes durante el desplazamiento y cuáles no lo son. }\end{array}$ \\
\hline & 0 & $\begin{array}{l}\text { No indicar todas las fuerzas que actúan sobre el mono. En caso de hacerlo, no distinguir cuáles } \\
\text { son conservativas y cuáles no lo son, ni cuáles son constantes durante el desplazamiento y cuáles } \\
\text { no lo son. }\end{array}$ \\
\hline \multirow[t]{3}{*}{$\begin{array}{l}\text { Suficiencia } \\
\text { del razonamiento }\end{array}$} & 2 & $\begin{array}{l}\text { Explicitar que, como todas las fuerzas que realizan trabajo son conservativas, se conserva la ener- } \\
\text { gía mecánica, y que, como todas las fuerzas que actúan son constantes, la aceleración resultante } \\
\text { también lo es, por lo que la cinemática del movimiento rectilíneo y uniformemente acelerado } \\
\text { es una vía sencilla de resolución. }\end{array}$ \\
\hline & 1 & Explicitar uno de los dos razonamientos anteriores. \\
\hline & 0 & No explicitar ninguno de los dos razonamientos anteriores. \\
\hline
\end{tabular}

Tabla 2.

Criterios específicos de valoración de la calidad

de los argumentos correspondientes a la situación problemática II

\begin{tabular}{|l|c|l|}
\hline \multicolumn{2}{|c|}{ Situación II } \\
\hline \multirow{2}{*}{$\begin{array}{l}\text { Conclusión } \\
\text { apropiada }\end{array}$} & 2 & $\begin{array}{l}\text { Proponer utilizar el principio de conservación de la energía mecánica y descartar la aplicación de } \\
\text { la segunda ley de Newton junto con la cinemática del movimiento rectilíneo y uniformemente } \\
\text { acelerado. }\end{array}$ \\
\cline { 2 - 3 } & 1 & $\begin{array}{l}\text { Proponer utilizar el principio de conservación de la energía mecánica y no descartar la aplicación } \\
\text { de la segunda ley de Newton junto a la cinemática del movimiento rectilíneo y uniformemente } \\
\text { acelerado. }\end{array}$ \\
\cline { 2 - 3 } & 0 & $\begin{array}{l}\text { Proponer utilizar otra vía de resolución distinta al principio de conservación de la energía me- } \\
\text { cánica o no proponer ninguna. }\end{array}$ \\
\hline
\end{tabular}




\begin{tabular}{|c|c|c|}
\hline \multirow{3}{*}{$\begin{array}{l}\text { Pertinencia } \\
\text { conceptual }\end{array}$} & 2 & $\begin{array}{l}\text { Considerar la posibilidad de aplicar el principio de conservación de la energía, así como la segun- } \\
\text { da ley de Newton y las leyes cinemáticas (con independencia de la propuesta final). }\end{array}$ \\
\hline & 1 & $\begin{array}{l}\text { Considerar la posibilidad de aplicar el principio de conservación de la energía o tener en cuenta } \\
\text { la segunda ley de Newton y las leyes cinemáticas (con independencia de la propuesta final). }\end{array}$ \\
\hline & 0 & No considerar la posibilidad de aplicar ninguna de las leyes o principios anteriores. \\
\hline \multirow{3}{*}{$\begin{array}{l}\text { Calidad } \\
\text { de la prueba }\end{array}$} & 2 & $\begin{array}{l}\text { Indicar todas las fuerzas que actúan sobre el mono. Distinguir cuáles son conservativas y cuáles } \\
\text { no lo son. Distinguir cuáles son constantes durante el desplazamiento y cuáles no lo son. }\end{array}$ \\
\hline & 1 & $\begin{array}{l}\text { Indicar todas las fuerzas que actúan sobre el mono. Distinguir cuáles son conservativas y cuáles } \\
\text { no lo son o distinguir cuáles son constantes durante el desplazamiento y cuáles no lo son. }\end{array}$ \\
\hline & 0 & $\begin{array}{l}\text { No indicar todas las fuerzas que actúan sobre el mono. En caso de hacerlo, no distinguir cuáles } \\
\text { son conservativas y cuáles no lo son, ni cuáles son constantes durante el desplazamiento y cuáles } \\
\text { no lo son. }\end{array}$ \\
\hline \multirow[t]{3}{*}{$\begin{array}{l}\text { Suficiencia } \\
\text { del razonamiento }\end{array}$} & 2 & $\begin{array}{l}\text { Explicitar que, como todas las fuerzas que realizan trabajo son conservativas, se conserva la ener- } \\
\text { gía mecánica, y que, como no todas las fuerzas que actúan son constantes (la elástica varía con } \\
\text { la posición), la aceleración resultante no es constante, por lo que la cinemática del movimiento } \\
\text { rectilíneo y uniformemente acelerado no es una vía adecuada. (Otras cinemáticas resultarían } \\
\text { más complejas). }\end{array}$ \\
\hline & 1 & Explicitar uno de los dos razonamientos anteriores. \\
\hline & 0 & No explicitar ninguno de los dos razonamientos anteriores. \\
\hline
\end{tabular}

Tabla 3.

Criterios específicos de valoración de la calidad de los argumentos correspondientes a la situación problemática III

\begin{tabular}{|c|c|c|}
\hline \multicolumn{3}{|r|}{ Situación III } \\
\hline \multirow{3}{*}{$\begin{array}{l}\text { Conclusión } \\
\text { apropiada }\end{array}$} & 2 & $\begin{array}{l}\text { Proponer utilizar el principio de conservación del momento lineal y el principio de conservación } \\
\text { del momento angular. }\end{array}$ \\
\hline & 1 & Proponer utilizar solo una de las dos vías posibles señalas en el nivel 2. \\
\hline & 0 & Proponer utilizar otra vía de resolución o no proponer ninguna. \\
\hline \multirow{3}{*}{$\begin{array}{l}\text { Pertinencia } \\
\text { conceptual }\end{array}$} & 2 & $\begin{array}{l}\text { Considerar la posibilidad de aplicar el principio conservación del momento lineal y el principio } \\
\text { de conservación del momento angular (con independencia de la propuesta final). }\end{array}$ \\
\hline & 1 & $\begin{array}{l}\text { Considerar la posibilidad de aplicar solo uno de los dos principios seńalados en el nivel anterior } \\
\text { (con independencia de la propuesta final). }\end{array}$ \\
\hline & 0 & No considerar la posibilidad de aplicar ninguna de las leyes o principios anteriores. \\
\hline \multirow{3}{*}{$\begin{array}{l}\text { Calidad } \\
\text { de la prueba }\end{array}$} & 2 & $\begin{array}{l}\text { Indicar todas las fuerzas que actúan sobre el bastón y la pelota de plastilina. Distinguir entre } \\
\text { fuerzas externas e internas al sistema. }\end{array}$ \\
\hline & 1 & $\begin{array}{l}\text { Indicar todas las fuerzas que actúan sobre el bastón y la pelota de plastilina. Explicitar solo cuáles } \\
\text { son externas o solo cuáles son internas. }\end{array}$ \\
\hline & 0 & $\begin{array}{l}\text { No indicar todas las fuerzas que actúan sobre el bastón y la pelota de plastilina. En caso de } \\
\text { hacerlo, no explicitar ni cuáles son internas ni cuáles son externas. }\end{array}$ \\
\hline \multirow{3}{*}{$\begin{array}{l}\text { Suficiencia } \\
\text { del razonamiento }\end{array}$} & 2 & $\begin{array}{l}\text { Explicitar que, como las fuerzas que actúan durante la colisión son internas, puesto que la nor- } \\
\text { mal y el peso se cancelan entre sí, se conserva el momento lineal, y que como el momento de las } \\
\text { fuerzas externas es nulo, se conservará el momento angular. }\end{array}$ \\
\hline & 1 & Explicitar uno de los dos razonamientos anteriores. \\
\hline & 0 & No explicitar ninguno de los dos razonamientos anteriores. \\
\hline
\end{tabular}


Tabla 4.

Criterios específicos de valoración de la calidad

de los argumentos correspondientes a la situación problemática IV

\begin{tabular}{|c|c|c|}
\hline \multicolumn{3}{|r|}{ Situación IV } \\
\hline \multirow{3}{*}{$\begin{array}{l}\text { Conclusión } \\
\text { apropiada }\end{array}$} & 2 & $\begin{array}{l}\text { Proponer utilizar el principio de conservación del momento angular y descartar la aplicación del } \\
\text { principio de conservación del momento lineal. }\end{array}$ \\
\hline & 1 & $\begin{array}{l}\text { Proponer utilizar el principio de conservación del momento angular y no descartar el principio } \\
\text { de conservación del momento angular. }\end{array}$ \\
\hline & 0 & $\begin{array}{l}\text { Proponer utilizar otra vía de resolución distinta a la conservación del momento angular o no } \\
\text { proponer ninguna. }\end{array}$ \\
\hline \multirow{3}{*}{$\begin{array}{l}\text { Pertinencia } \\
\text { conceptual }\end{array}$} & 2 & $\begin{array}{l}\text { Considerar la posibilidad de aplicar el principio de conservación del momento lineal y el princi- } \\
\text { pio de conservación del momento angular (con independencia de la propuesta final). }\end{array}$ \\
\hline & 1 & $\begin{array}{l}\text { Considerar la posibilidad de aplicar solo uno de los dos principios señalados en el nivel anterior } \\
\text { (con independencia de la propuesta final). }\end{array}$ \\
\hline & 0 & No considerar la posibilidad de aplicar ninguna de las leyes o principios anteriores. \\
\hline \multirow{3}{*}{$\begin{array}{l}\text { Calidad } \\
\text { de la prueba }\end{array}$} & 2 & $\begin{array}{l}\text { Indicar todas las fuerzas que actúan sobre la puerta y la pelota de plastilina. Distinguir entre } \\
\text { fuerzas externas e internas al sistema. }\end{array}$ \\
\hline & 1 & $\begin{array}{l}\text { Indicar todas las fuerzas que actúan sobre la puerta y la pelota de plastilina. Explicitar solo } \\
\text { cuáles son externas o solo cuáles son internas. }\end{array}$ \\
\hline & 0 & $\begin{array}{l}\text { No indicar todas las fuerzas que actúan sobre la puerta y la pelota de plastilina. En caso de } \\
\text { hacerlo, no explicitar ni cuáles son internas ni cuáles son externas. }\end{array}$ \\
\hline \multirow{3}{*}{$\begin{array}{l}\text { Suficiencia } \\
\text { del razonamiento }\end{array}$} & 2 & $\begin{array}{l}\text { Explicitar que, como consecuencia de las bisagras que sujetan la puerta, las fuerzas externas que } \\
\text { actúan durante la colisión no son despreciables frente a las fuerzas internas y, en consecuencia, } \\
\text { no se conserva el momento lineal, y explicitar que como el momento de las fuerzas externas es } \\
\text { nulo, puesto que las fuerzas externas se aplican en el eje de rotación, se conservará el momento } \\
\text { angular con respecto al eje de rotación. }\end{array}$ \\
\hline & 1 & Explicitar uno de los dos razonamientos anteriores. \\
\hline & 0 & No explicitar ninguno de los dos razonamientos anteriores. \\
\hline
\end{tabular}

Los datos han sido recogidos en situación de examen una vez finalizado el primer cuatrimestre, de manera que quede garantizado el interés de los estudiantes por la tarea. En ese momento ya se había impartido en todos los grupos el conocimiento básico necesario de los conceptos, leyes y principios de la mecánica. La resolución por escrito de problemas de física ha sido, en este estudio, el principal instrumento utilizado como fuente de datos (Rivard, 1994). Entendemos que al ser este un contexto de menor presión resulta más adecuado que una sesión oral para que los estudiantes expliquen razonadamente las vías de resolución. Por otro lado, los científicos deben ser capaces de compartir los resultados de sus investigaciones por medio del lenguaje escrito y deben saber escribir de tal manera que refleje los estándares y las normas de la comunidad científica (Shanahan, 2004). Sin embargo, cuando han existido dudas en la categorización de las respuestas, también se han utilizado datos de grabaciones de entrevistas realizadas a veinte estudiantes, elegidos entre aquellos que han conseguido un "nivel medio» en el aprendizaje de la física y que, de forma voluntaria, han aclarado oralmente sus planteamientos. Por razones de espacio estas entrevistas no se transcriben en este trabajo, aunque debe entenderse que las ideas en ellas expresadas son coincidentes con los ejemplos que incluimos en el siguiente apartado, extraídas de las resoluciones escritas. 


\section{RESULTADOS}

Las respuestas de los estudiantes han sido analizadas por dos de los autores de la investigación. El grado de concordancia se ha valorado con la Kappa de Cohen y, tal y como se puede observar en la tabla 5, se ha logrado un nivel de acuerdo entre «sustancial» y «casi perfecto» según la escala de Landis y Koch (1977).

Tabla 5 .

Grado de concordancia alcanzado en las puntuaciones

\begin{tabular}{|l|c|c|c|c|}
\hline \multicolumn{5}{|c|}{ Kappa de Cohen } \\
\hline \multicolumn{1}{|c|}{ Aspecto valorado } & Situación I & Situación II & Situación III & Situación IV \\
\hline Conclusión apropiada & 0,803 & 0,861 & 0,851 & 1 \\
\hline Pertinencia conceptual & 1 & 0,888 & 0,851 & 0,712 \\
\hline Calidad de la prueba & 0,720 & 0,628 & 0,637 & 0,624 \\
\hline Suficiencia del razonamiento & 0,736 & 0,724 & 0,837 & 1 \\
\hline
\end{tabular}

Así mismo, es necesario señalar que, tal y como era previsible teniendo en cuenta las características de los dos grupos analizados, no se han producido diferencias significativas entre los resultados obtenidos por los estudiantes de ambos grupos, por lo que hemos considerado una única muestra de estudiantes. En la tabla 6 y en el gráfico 1 recogemos los resultados obtenidos en las situaciones I y II, para las que se han obtenido 153 respuestas, y en las situaciones III y IV, para las que la muestra se ha reducido a un total de 144 estudiantes. En ellas se reflejan las puntuaciones promedio obtenidas por los estudiantes, en cada situación, para cada uno de los cuatro aspectos valorados sobre un máximo de 2 (conclusión apropiada, pertinencia conceptual, calidad de la prueba y suficiencia del razonamiento), así como la puntuación total sobre un máximo de 8 .

Tabla 6.

Puntuaciones promedio obtenidas por los estudiantes

\begin{tabular}{|c|c|c|c|c|c|}
\hline Situación & $\begin{array}{c}\text { Conclusión } \\
\text { apropiada } \\
\text { (máximo 2) }\end{array}$ & $\begin{array}{c}\text { Pertinencia } \\
\text { conceptual } \\
\text { (máximo 2) }\end{array}$ & $\begin{array}{c}\text { Calidad } \\
\text { de la prueba } \\
\text { (máximo 2) }\end{array}$ & $\begin{array}{c}\text { Suficiencia } \\
\text { del razonamiento } \\
\text { (máximo 2) }\end{array}$ & $\begin{array}{c}\text { Total } \\
\text { (máximo 8) }\end{array}$ \\
\hline$I(\mathrm{~N}=153)$ & 1,5 & 1,6 & 0,7 & 0,6 & 4,5 \\
\hline$I I(\mathrm{~N}=153)$ & 1,0 & 1,2 & 0,3 & 0,3 & 1,8 \\
\hline$I I I(\mathrm{~N}=144)$ & 0,6 & 0,6 & 0,2 & 0,3 & 1,2 \\
\hline$I V(\mathrm{~N}=144)$ & 0,3 & 0,5 & 0,2 & 0,2 & 2,7 \\
\hline
\end{tabular}




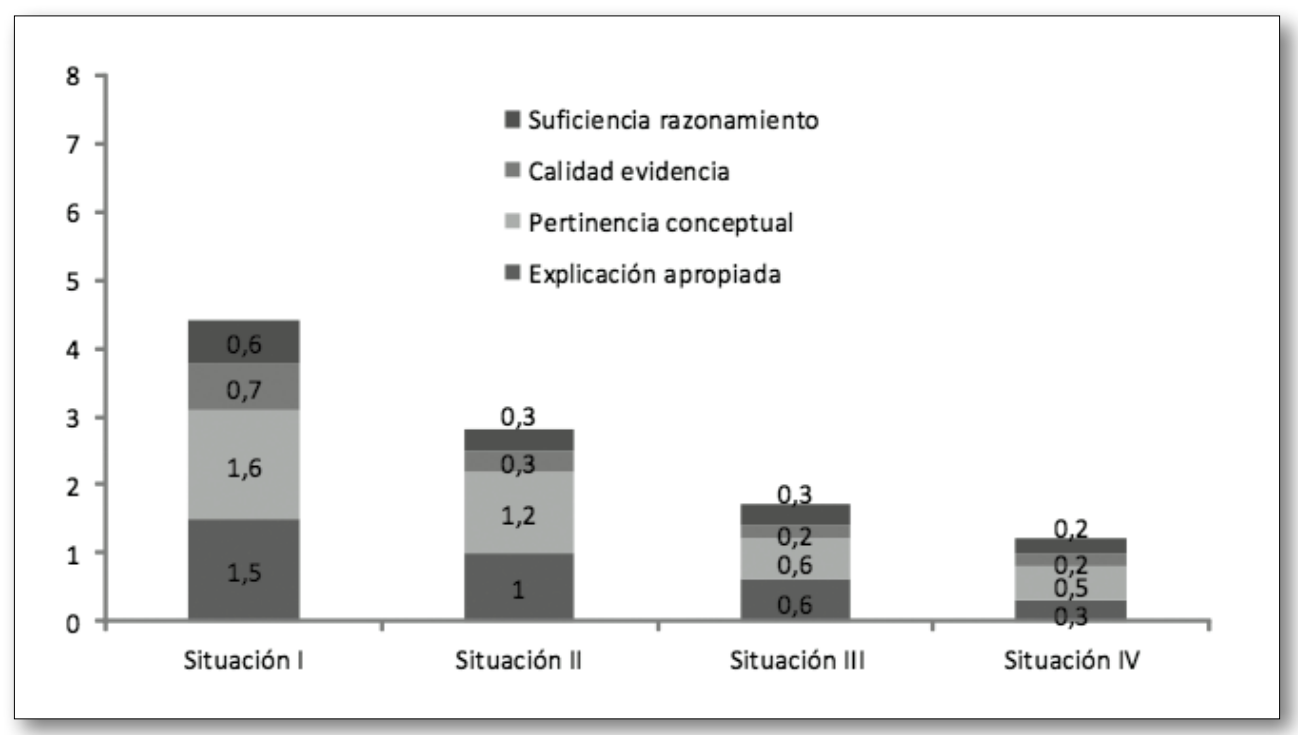

Gráfico 1. Puntuaciones promedio obtenidas por los estudiantes.

A la vista de los resultados, se observa que la calidad de los argumentos expuestos por los estudiantes en su análisis de las situaciones III y IV es claramente inferior a la correspondiente a las situaciones I y II. Las puntuaciones totales obtenidas han sido de 4,5 (situación I), 2,7 (situación II), 1,8 (situación III) y 1,2 (situación IV). Si bien es cierto que el contexto de aplicación a las colisiones en el ámbito del sólido rígido supera en dificultad el estudio de la caída libre o amortiguada, no consideramos que este fuerte descenso deba interpretarse únicamente como consecuencia de una deficiencia conceptual.

En las situaciones I y II, observamos que tanto las conclusiones como la pertinencia conceptual aportadas son, en general, aceptables, con puntuaciones superiores a 1, siendo estas más elevadas en el caso de la caída libre que en el caso del descenso bajo el efecto de la fuerza elástica. Este resultado parece del todo lógico teniendo en cuenta lo familiarizados que se encuentran los estudiantes con el estudio de la caída libre y, en menor medida, con el movimiento en el que interviene la fuerza elástica. Sin embargo, el fracaso se incrementa, especialmente en la situación II, cuando se trata de identificar las interacciones que actúan sobre el mono, si son constantes, conservativas o no conservativas (calidad de la prueba) y cuando, como consecuencia de ello, tienen que razonar qué leyes o principios son aplicables en esa situación (suficiencia del razonamiento).

En las situaciones III y IV, ningún aspecto alcanza puntuaciones superiores a 0,6 sobre 2, destacando, nuevamente, los pobres resultados obtenidos en la calidad de las pruebas empleadas y en la suficiencia de los razonamientos emitidos, que alcanzan, como máximo, la puntuación de 0,3. Las conclusiones a las que llegan y la pertinencia de las leyes y principios que consideran en sus análisis (pertinencia conceptual) son, así mismo, muy deficientes en las dos situaciones, especialmente en la situación IV.

A modo de ejemplo, en primer lugar, incluimos para cada una de las cuatro situaciones un argumento de elevado nivel emitido por diversos estudiantes. Todos estos argumentos han sido valorados con puntuación total de 8 , en consonancia con lo establecido en los criterios específicos de las tablas 1 a 4 . En los ejemplos se presentan extractos de los escritos de los estudiantes, recogiendo solo los aspectos más representativos de aquello que queremos resaltar y omitiendo la nomenclatura matemática que pudieran haber utilizado los estudiantes en sus respuestas.

Ander-Situación I: «Si despreciamos el rozamiento, la única fuerza que interviene en la caída del mono es su peso, que es constante y, entonces, la aceleración es constante y el movimiento es MRUA. 
Por eso, la ley de Newton y las fórmulas de esa cinemática nos sirven para calcular la velocidad. El peso es conservativo, por lo que también podemos usar el principio de conservación de la energía».

Olatz-Situación II: «Se podrían utilizar la ley de Newton y las fórmulas de cinemática, pero como la aceleración no es constante porque la fuerza elástica cambia según cae, el problema se complicaría. Por eso, el principio de conservación de la energía es más fácil y se puede usar porque el peso y la fuerza elástica son conservativas (aquí también depreciamos el rozamiento con el aire)».

Iker-Situación III: «El choque se produce en una pista sin rozamiento y el peso y la normal se compensan, además de que son despreciables frente a las fuerzas internas mientras dura el choque. Como el sumatorio de fuerzas externas es cero, se puede aplicar la conservación del momento lineal para sacar la velocidad de salida. Como tampoco hay momento de las fuerzas externas, con la conservación del momento angular podemos sacar la velocidad angular».

Joana-Situación IV: «En este caso las fuerzas que en el momento del choque hacen las bisagras sobre la puerta no son despreciables frente a las fuerzas internas (bola-puerta). Por eso, las fuerzas externas no son cero y no podemos usar la conservación del momento lineal. Pero sí la conservación del momento angular, porque el momento de esas fuerzas que se aplican en el eje es cero».

Sin embargo, los argumentos que acabamos de señalar son una excepción. Atendiendo a la calidad de la prueba empleada, muchos estudiantes no se plantean la elección de una ley o principio basado en las características de las interacciones. No aportan pruebas que sustenten la conclusión. Veamos algunos casos:

Garazi-Situación I: «Como es una caída libre la cinemática del MRUA nos servirá para calcular la velocidad».

Comentario: no se cita el peso como la interacción que produce una aceleración constante, no se aporta un mecanismo causal.

Roberto-Situación II: «Desde el punto de vista de la energía, podríamos decir que, en la posición inicial, hay energía potencial gravitatoria y que cuando cae se va convirtiendo en energía cinética y en energía potencial elástica. Así podría calcular la velocidad. Si quisiera calcular por dinámica y por cinemática, sería más difícil».

Comentario: no se menciona el carácter conservativo de las fuerzas ni el hecho de que la fuerza elástica varíe con la posición, que es, en definitiva, la causa de que la vía cinemática le resulte más difícil.

Jon-Situación III: «Podemos aplicar las fórmulas de los choques inelásticos, o sea, la conservación del momento lineal».

Ibon-Situación IV: «Después del choque la puerta y la bola solo giran, por lo que hay que aplicar la conservación del momento angular».

Comentario: no se mencionan las interacciones y se asocian memorísticamente, como una receta, los choques con la conservación del momento lineal para la traslación y la conservación del momento angular para la rotación.

En otras ocasiones se ha detectado que determinados estudiantes no distinguen entre lo que son pruebas relevantes o irrelevantes cuando construyen un argumento. En ninguno de los siguientes ejemplos se aporta prueba alguna que sea relevante con respecto a la aplicabilidad del principio propuesto para la resolución de la situación:

Sergio-Situación I: «La razón que me ha llevado a aplicar la conservación de la energía es que aparecen la altura y la velocidad y en el principio de conservación de la energía están muy relacionadas».

Endika-Situación III: «He elegido la conservación del momento lineal porque con los datos que me dan pienso que es un camino adecuado. Sé que el bastón tenía velocidad nula y que la bola llega con velocidad v, por eso me sirve la conservación de $\mathrm{P}_{»}$. 
Finalmente, para la elección de la aplicación de una ley o principio, a menudo, los estudiantes no razonan suficientemente la relación entre las pruebas y las conclusiones. Lo comprobamos en los ejemplos que presentamos a continuación:

Andrea-Situación II (también utilizado para la Situación I): «Se puede aplicar la conservación de la energía porque no hay ninguna fuerza externa, es decir, las fuerzas externas son nulas».

Comentario: no es esta la condición que ha de cumplirse para que se conserve la energía.

Mariana-Situación IV: «La fuerza que la bola hace a la puerta en el choque es una fuerza interna que se compensa con la que la puerta hace a la pelota. Entonces, como el sumatorio de fuerzas externas es cero, en este caso también se conserva el momento lineal».

Comentario: no se identifican las fuerzas externas, no despreciables, que durante la colisión las bisagras ejercen sobre la puerta impidiendo su traslación.

\section{CONCLUSIONES}

Hemos analizado en este trabajo la calidad de los argumentos utilizados por los estudiantes universitarios que siguen una enseñanza tradicional en primer curso de Ingeniería cuando, ante una situación problemática de física, deben optar por la estrategia o vía de resolución más adecuada. Hemos valorado lo apropiado de sus conclusiones, la pertinencia de las leyes y los principios que han tenido en cuenta para llegar a una conclusión (pertinencia conceptual), la calidad de las pruebas manejadas y, con especial atención, si sus conclusiones se encuentran validadas por la coherencia de sus razonamientos (suficiencia del razonamiento).

Los resultados de estas valoraciones nos indican que solo la puntuación total correspondiente a la situación I ha superado la mitad de los puntos posibles (4,5 sobre un máximo de 8 ). Las deficiencias más destacables han surgido ligadas a la ausencia de pruebas que sustenten la conclusión y al hecho de que, cuando se consideran estas pruebas, a menudo no se razona suficientemente la relación entre ellas y las conclusiones, es decir, entre las características de las interacciones y la elección de las leyes y principios aplicables en la resolución. Muchas de las deficiencias detectadas en la argumentación han sido fallos por omisión: los estudiantes no han aportado aspectos que los investigadores consideran que se deben incluir. Los estudiantes también tienen dificultades en distinguir entre lo que son pruebas relevantes e irrelevantes cuando escriben un argumento.

Estos resultados son coherentes con los obtenidos por otros investigadores que indican que los estudiantes suelen tener grandes dificultades con diversos aspectos de la argumentación en ciencias (Jiménez-Aleixandre y Erduran, 2007; Osborne et al., 2004). Muchos estudiantes no comprenden en qué consiste un buen argumento en ciencia (McNeill y Krajcik, 2007; Sandoval y Reiser, 2004), por lo que tienden a dar explicaciones que son vagas e insuficientes o dan simplemente una descripción de lo que observan sin aportar un mecanismo causal de la cuestión que se quiere investigar. La investigación empírica también indica que los estudiantes, en muchas ocasiones, no razonan adecuadamente la relación entre las pruebas y las conclusiones (Kuhn y Reiser, 2005; McNeill y Krajcik, 2007).

Por todo ello, tal y como señala Jiménez-Aleixandre (2007), la inclusión de la argumentación en las clases de ciencias no debe plantearse como un objetivo desconectado del aprendizaje de la ciencia. Por el contrario, la argumentación forma parte de los objetivos de la ciencia constructivista en el aula, en la que el papel de los estudiantes es el de generadores de conocimiento, mientras que el de los profesores se corresponde con el de orientadores que guían la investigación, y todo ello planteado como una actividad o proceso social.

Desde esta perspectiva, un gran número de investigadores en el ámbito de la educación defienden que los estudiantes necesitan disponer de más oportunidades para aprender cómo la comunidad cientí- 
fica hace uso de los argumentos para construir conocimiento y los criterios que determinan qué cuenta como un buen argumento en ciencia (Driver et al., 2000; Duschl y Osborne, 2002; Kuhn y Reiser, 2005; Newton et al., 1999).

En el ámbito de la resolución de problemas en el que se ha centrado este estudio, consideramos que el modelo de resolución de problemas como investigación guiada reúne las características adecuadas para promover la argumentación en las clases de Física en la universidad (Ceberio et al., 2008; Guisasola et al., 2006a; Guisasola et al., 2006b; Guisasola et al., 2011).

\section{REFERENCIAS BIBLIOGRÁFICAS}

Abell, S. K.; Anderson, G. y Chezem, J. (2000). Science as argument and explanation: Exploring concepts of sound in third grade. En J. Minstrell y E. H. Van Zee (eds.). Inquiry into inquiry learning and teaching in science. Washington, DC: American Association for the Advancement of Science, pp. 100-119.

BeLl, P. (2004). Promoting students' argument construction and collaborative debate in the science classroom. En M. Linn, E. A. Davis y P. Bell (eds.). Internet environments for science education. Mahwah, NJ: Erlbaum.

Ceberio, M.; Guisasola J. y Almudí J.M. (2008). ¿Cuáles son las innovaciones didácticas que propone la investigación en resolución de problemas de física y qué resultados alcanzan? Enseñanza de las Ciencias, 26 (3), pp. 419-430.

Chalmers, A.F. (2000). ¿Qué es esa cosa llamada ciencia? Siglo XXI: Madrid.

Clark, D. y Sampson, V. (2006). Personally-seeded discussions to scaffold online argumentation. International Journal of Science Education, 29 (3), pp. 253-277.

http://dx.doi.org/10.1080/09500690600560944

Driver, R.; Newton, P. y Osborne, J. (2000). Establishing the norms of scientific argumentation in classrooms. Science Education, 84 (3), pp. 287-313.

http://dx.doi.org/10.1002/(SICI)1098-237X(200005)84:3<287::AID-SCE1>3.3.CO;2-1

Duschl, R. (2000). Making explicit the nature of science. En R. Millar, J. Leach, y J. Osborne (eds.). Improving science education: Contributions from research. Philadelphia, PA: Open University Press, pp. 187-206.

Duschl, R. (2008). Science education in three-part harmony: Balancing conceptual, epistemic, and social learning goals. Review of Research in Education, 32, pp. 268-291.

http://dx.doi.org/10.3102/0091732X07309371

Duschl, R. y Osborne, J. (2002). Supporting and promoting argumentation discourse. Studies in Science Education, 38, pp. 39-72.

http://dx.doi.org/10.1080/03057260208560187

Eemeren, F. H. van y Grootendorst, R. (2004). A systematic theory of argumentation. The pragmadialected approach. Cambridge: Cambridge University Press.

Erduran, S.; Simon, S. y Osborne, J. (2004). TAPing into argumentation: Developments in the application of Toulmin's argument pattern for studying science discourse. Science Education, 88, pp. 915-933.

http://dx.doi.org/10.1002/sce.20012

Guisasola, J.; Ceberio, M. y Zubimendi J.L. (2006a). University Students' Strategies for Constructing Hypothesis when Tackling Paper-and-Pencil Tasks in Physics. Research In Science Education, 36 (3), pp. 163-186.

http://dx.doi.org/10.1007/s11165-005-9000-7 
Guisasola, J.; Ceberio, M.; Almudí, J.M. y Zubimendi, J.L. (2006b). La enseñanza de problemas de Fisica en primer curso de universidad. De explicar problemas a guiar su resolución. Barcelona: Editorial Octaedro.

Guisasola, J.; Ceberio, M.; Almudi, J.M. y Zubimendi, J.L. (2011). La resolución de problemas basada en el desarrollo de investigaciones guiadas en cursos introductorios de física universitaria. Enseñanza de las Ciencias, 29 (3), pp. 439-452.

Jiménez-Aleixandre, M. P. (2007). Designing Argumentation Learning Environments. En S. Erduran y M. P. Jiménez-Aleixandre (eds.). Argumentation in science education: perspectives from classroombased research. Dordrecht, The Netherlands: Springer, pp. 91-116. http://dx.doi.org/10.1007/978-1-4020-6670-2_5

Jiménez-Aleixandre, M. P. y Erduran, S. (2007). Argumentation in Science Education: An Overview. En S. Erduran y M. P. Jiménez-Aleixandre (eds.). Argumentation in science education: perspectives from classroom-based research. Dordrecht, The Netherlands: Springer, pp. 3-28. http://dx.doi.org/10.1007/978-1-4020-6670-2_1

Jiménez-Aleixandre, M.; Rodríguez, M. y Duschl, R.A. (2000). «Doing the lesson» or «doing science»: Argument in high school genetics. Science Education, 84 (6), pp. 757-792. http://dx.doi.org/10.1002/1098-237X(200011)84:6<757::AID-SCE5>3.0.CO;2-F

Kenyon, L.; Kuhn, L. y Reiser, B.J. (2006). Using students' epistemologies of science to guide the practice of argumentation. En S.A. Barab, K.E. Hay y T.D. Hickey (eds.). Proceedings of the 7thInternational conference of the Learning Science. Mahwah, NJ: Lawrence Erlbaum, pp. 321-327.

Kunn, D. (2005). Education for thinking. Cambridge, MA: Harvard University Press.

Kunn, L. y ReIser, B. (2005). Students constructing and defending evidence-based scientific explanations. Paper presented at the annual meeting of the National Association for Research in Science Teaching, Dallas, TX.

Kuhn, L. y ReIser, B. (2006). Structuring activities to foster argumentative discourse. Paper presented at the annual meeting of the American Educational Research Association, San Francisco, CA.

Kunn, D. y Udell, W. (2003). The development of argument skills. Child Development, 74 (5), pp. 1245-1260.

http://dx.doi.org/10.1111/1467-8624.00605

Landis, J. R. y Косн, G. G. (1977). The measurement of observer agreement for categorical data. Biometric, 33, pp. 159-174.

http://dx.doi.org/10.2307/2529310

Lawson, A. (2002). Sound and faulty arguments generated by preservice biology teachers when testing hypotheses involving unobservable entities. Journal of Research in Science Teaching, 39 (3), pp. 237-252.

http://dx.doi.org/10.1002/tea.10019

Martínez Torregrosa, J.; Gil, D.; Becerra, C. y Guisasola J. (2005). ¿Podemos mejorar la enseñanza de la resolución de problemas de «lápiz y papel» en las aulas de Física y Química? Educación quimica, 16 (2), pp. 230-245.

MCNeILL, K.L. y KRAJCIK, J. (2007). Middle school students' use of appropriate and inappropriate evidence in writing scientific explanations. En M. Lovett, y P. Shah (eds.). Thinking with data: The proceedings of 33rd Carnegie symposium on cognition. Mahwah, NJ: Erlbaum.

Newton, P.; Driver, R. y Osborne, J. (1999). The place of argumentation in the pedagogy of school science. International Journal of Science Education, 21 (5), pp. 553-576.

http://dx.doi.org/10.1080/095006999290570 
Osborne, J.; Erduran, S. y Simon, S. (2004). Enhancing the quality of argumentation in science classrooms. Journal of Research in Science Teaching, 41 (10), pp. 994-1020.

http://dx.doi.org/10.1002/tea.20035

Pellegrino, J.; Chudowsky, N. y Glaser, R. (2001). Knowing what student know. Washington, DC: National Academy Press. Disponible en línea: http://www.nap.edu.

Perelman, C. y Olbrechts-Tyteca, L. (1958). Traité de l'argumentation. La nouvelle rhétorique. Bruxelles: Éditions de l'Université de Bruxelles. (The new rhetoric: A treatise on argumentation. Notre Dame, IN: University of Notre Dame Press, 1969).

RIVARD, L.P. (1994). A review of writing to learn science: implications for practice and research. Journal of Research in Science Teaching, 31, pp. 969-983.

http://dx.doi.org/10.1002/tea.3660310910

Sampson, V. y Clark, D. (2008). Assessment of the ways students generate arguments in science education: Current perspectives and recommendations for future directions. Science Education, 92, pp. 447-472.

http://dx.doi.org/10.1002/sce.20276

Sampson, V. y Clark, D. (2009). The Impact of Collaboration on the Outcomes of Scientific Argumentation. Science Education, 93, pp. 448-484.

http://dx.doi.org/10.1002/sce.20306

Sampson, V.; Grooms, J. y Walker, J.P. (2011). Argument-Driven Inquiry as a Way to Help Students Learn How to Participate in Scientific Argumentation and Craft Written Arguments: An Exploratory Study. Science Education, 95, pp. 217-257. http://dx.doi.org/10.1002/sce.20421

Sandoval, W.A. y Millwood, K.A. (2005). The quality of students' use of evidence in written scientific explanations. Cognition and Instruction, 23 (1), pp. 23-55. http://dx.doi.org/10.1207/s1532690xci2301_2

SANDOval, W.A. y Reiser, B.J. (2004). Explanation-driven inquiry: Integrating conceptual and epistemic scaffolds for scientific inquiry. Science Education, 88, pp. 345-372. http://dx.doi.org/10.1002/sce.10130

SAwyer, R. (2006). The Cambridge handbook of the learning sciences. New York: Cambridge University Press.

Shanahan, C. (2004). Better textbooks, better readers and writers. En W. Saul (ed.). Crossing borders in literacy and science instruction: Perspectives on theory and practice. Arlington, VA: NSTA Press.

Toulmin, S. (1958). The uses of argument. Cambridge: Cambridge University Press.

Walton, D., Reed, C. y Macagno, F. (2008). Argumentation Schemes, New York: Cambridge University Press.

Zohar, A. y Nemet, F. (2002). Fostering students' knowledge and argumentation skills through dilemmas in human genetics. Journal of Research in Science Teaching, 39 (1), pp. 35-62.

http://dx.doi.org/10.1002/tea.10008 


\section{Analysis of the arguments created by students in introductory courses of university Physics when tackling problematic situations}

Mikel Ceberio, José Manuel Almudí, José Luis Zubimendi

Departamento de Física Aplicada I, Euskal Herriko Unibertsitatea (UPV/EHU)

mikel.ceberio@ehu.es; josemanuel.almudi@ehu.es; jl.zubimendi@ehu.es

In the last years, an increasing number of research are focusing on the analysis of the argumentation in science lessons. The analysis of the arguments made by the students can provide important information about the understanding of the scientific content (e.g., theories, laws, and principles of physics), the scientific reasoning of students, epistemological aspects (e.g., what counts as justification in science) and about the ability of students to communicate and justify ideas.

On the other hand, when students face 'pencil and paper' problems to come to a resolution, it is necessary to identify the laws and principles of physics applicable in that context. It is also necessary when tackling a problematic situation, assessing possible alternative ways of resolution. The choice of one or another goes through a proper justification on the basis of evidence.

In this article, we intend to analyze the quality of the arguments used by the students who follow a traditional teaching first course of engineering when facing problematic situations of physics and they must choose by the strategy or most appropriate approach. We try to verify if their conclusions, in this sense, are certainly validated by the consistency of their reasoning on the basis of the analysis of the characteristics concerning the situation interactions, which will constitute the evidence of the argument.

Our analysis is based on the model proposed by Sampson and Clark which, at the same time, was inspired by Toulmins model and is consistent with other researchers in science education. In this model, it is considered that a scientific argument consists of three interrelated components: the conclusion or explanation, evidence and reasoning.

In our work, we have specifically focused the attention on the main aspects that the literature uses to evaluate the quality of the written arguments created by the students, namely: a) the appropriateness of the conclusion, which should be correct, b) the relevance of conceptual references considered in the argument, which must be related to the laws and principles of matter, c) the quality of the evidence, which must be adequate and sufficient and d) the sufficiency of the reasoning, which should justify that the evidence supports the conclusion.

Research was conducted by 153 students from first course in Engineering Degree at the University of the Basque Country. To collect data, we have asked students to argue in a justified manner, individually and in writing, the path of resolution they consider most appropriate to solve a particular problematic situation.

Most noteworthy deficiencies have been linked to the lack of evidence supporting the conclusion and with the fact that quite often, when considering evidence, they do not properly relate the relationship between these evidence and the conclusions obtained, (between the characteristics of the interactions and the choice of laws and principles applicable to the resolution). Many of the deficiencies identified in the argument have been failures by default: students have not described what researchers consider should be included. Students also have difficulties in distinguishing between what is relevant and irrelevant evidence when writing an argument.

A large number of researchers in the field of education argue that students need to have more opportunities to learn how the scientific community uses the arguments to build knowledge and the criteria determining what counts as a good argument in science.

In the field of problem solving in which this study focuses, we consider that the Inquiry-Based Problem Solving model meets the appropriate characteristics to promote the argumentation in physics in university classes. 